\title{
The cold neutron tomography set-up at SINQ
}

\author{
T. Materna ${ }^{\mathrm{a}, *}$, S. Baechler ${ }^{\mathrm{b}}$, J. Jolie $^{\mathrm{a}}$, B. Masschaele ${ }^{\mathrm{c}}$, \\ M. Dierick ${ }^{\mathrm{c}}$, N. Kardjilov ${ }^{\mathrm{d}}$ \\ a Institut für Kernphysik, Universität zu Köln, Köln D-50937, Germany \\ ${ }^{\mathrm{b}}$ Physics Department, University of Fribourg, Fribourg CH-1700, Switzerland \\ ${ }^{\mathrm{c}}$ Vakgroep Subatomaire en Stralingsfysika, Ghent University, Gent B-9000, Belgium \\ ${ }^{\mathrm{d}}$ Fakultät für Physik E21, Technische Universität München, Garching D-85747, Germany
}

\begin{abstract}
The cold neutron tomography station operated at SINQ (Paul Scherrer Institute, Switzerland) is reviewed. The high neutron flux together with a set-up based on a scintillator screen and a CCD camera yielded fast and effective results with resolution down to $250 \mu \mathrm{m}$ : tomography of small samples (up to $2 \mathrm{~cm}$ large) could be performed in less than an hour. The use of a velocity selector improved the contrast discrimination and allows dichromatic tomography. The station is moving to the new research reactor FRM-II (Garching, Germany) where its performances will be increased by the availability of a 5-times-higher cold neutron flux.
\end{abstract}

PACS: $87.59 . \mathrm{F}$

Keywords: Neutron tomography

\section{Introduction}

Imaging with neutrons offers unique opportunities: it permits inspection of hydrogenous materials, which are transparent for X-rays; it allows distinguishing between elements close in the Mendeleyev table, which would have the same contrast with X-rays; one can image radioactive waste within a lead container, because heavy elements are quite transparent to neutrons; finally,

\footnotetext{
*Corresponding author. Tel.: + 49-221-470-36-22; fax: +49221-470-51-68.

E-mail address: thomas.materna@ikp.uni-koeln.de (T. Materna).
}

one can distinguish between isotopes. All these properties arise because neutrons interact by scattering or absorption with atomic nuclei while $\mathrm{X}$-rays interact with the electrons surrounding a nucleus.

The main reason to use cold neutrons is to improve the image contrast. Far from resonances, the cross-section for neutron capture is inversely proportional to the neutron velocity, or using the particle-wave duality, is proportional to its wavelength. Thus comparing thermal $(1.8 \AA)$ and cold neutrons $(5.5 \AA)$, the image contrast obtained with cold neutrons is generally about the cube of that with thermal neutrons.

Tomography provides information on the inner structure of a sample by taking radiographs 
at different orientations of the sample and reconstructing off-line all data in a virtual 3D object related to the neutron cross-section density. This virtual object can be manipulated, sliced open, quantified or compared to a reference object. However, tomography requires a high flux of neutrons to perform the object inspection in a reasonable time (few hours or less). For that reason, only a few neutron tomography set-ups exist in the world. The situation in Europe was, for example, reviewed in Ref. [1].

The current standard detection system to record a neutron radiograph for tomography purposes consists of a fluorescent screen sensitive to neutrons and a high resolution CCD camera. This technique is fast, cheap and efficient but also requires a low level of gamma background, the fluorescent screen being sensitive to X-and gamma rays. Cold neutrons, transported from the neutron cold source to the experiment by a neutron beam guide, are free from gamma background because neutron guides are bent in the first section for this purpose.

Another benefit of imaging with cold neutrons is that the Bragg cut-offs of many solids are in the cold neutron wavelength range. At a so-called Bragg cut-off wavelength, the neutron attenuation coefficient drops drastically. The reason is that in polycrystalline material, only neutrons with wavelengths shorter than 2 times the lattice distance of available crystal planes can satisfy the Bragg law and diffract or in other words be attenuated by diffraction. If the drop in attenuation is important and one can monochromatize the beam and select its wavelength, this behavior can be exploited to change the material contrast. Indeed, if one takes 2 radiographs, one with a wavelength just below the Bragg cut-off of a selected element, and one with a wavelength just above it, the ratio will be sensitive to this element only. On the other hand, if we select two wavelengths where the attenuation coefficient is equal, the element disappears in the ratio of the two radiographs. Finally, the drop at Bragg cut-off is useful to perform dichromatic tomography. This is similar to what one can do with $\mathrm{X}$-rays using rise in attenuation at $\mathrm{K}$-edge energy [2].

\section{Installation at SINQ}

\subsection{PGA beam line}

The Swiss spallation source SINQ of the Paul Scherrer Institute provides a continuous high flux $\left(10^{14} \mathrm{n} / \mathrm{cm}^{2} / \mathrm{s}\right)$ of neutrons [3] by impinging a large current $(1.8 \mathrm{~mA})$ of $590 \mathrm{MeV}$-protons on a lead target. This large-scale installation contained up to last year three permanent positions for neutron imaging inspection: a radiographic and tomographic station with thermal neutrons (NEUTRA) [4], a radiographic station with cold neutrons (NCR) [5] and the cold neutron tomography station reviewed in this paper. We operated this station alternatively with a spectroscopic facility for prompt gamma-ray activation at the end of a 63-m-long beam guide. The neutron wavelengths ranged from about 2 to $8 \AA$ with an effective wavelength of $5.5 \AA$, the beam size was $2 \mathrm{~cm} \times 5 \mathrm{~cm}$ and the flux was $1.8 \times 10^{8} \mathrm{n} / \mathrm{cm}^{2} / \mathrm{s}$. The method of transportation in the guide, by total reflection on the internal surface, yields a beam divergence of about $1.1^{\circ}$. The divergence is wavelength dependent and the value of $1.1^{\circ}$ was calculated for $5.5 \AA$.

\subsection{Standard set-up}

The set-up consists of the detection system and a $x y z$ translation-rotation table supporting the sample, motorized for rotation and for the vertical and horizontal direction perpendicular to the beam. This allows scanning samples larger than the beam size. The detection system comprises of a fast 12 -bit CCD camera with $1280 \times 1024$ pixels of $6.7 \mu \mathrm{m} \times 6.7 \mu \mathrm{m}$ size and a readout time of 12 full frames per second, and a scintillator screen made of $\mathrm{ZnS}(\mathrm{Ag}) /{ }^{6} \mathrm{LiF}$. Neutrons passing through the sample and arriving in the scintillator create alpha particles by reaction with ${ }^{6} \mathrm{Li}$ and these alphas produce blue light scintillations by reaction with $\mathrm{ZnS}(\mathrm{Ag})$. The distribution of these scintillations is imaged on the CCD by means of a standard objective lens. To keep the camera out of the direct beam, the camera observes the scintillator screen by reflection in a silver-free mirror. A lead glass placed between the objective and the mirror protects the CCD chip from gamma rays emitted 
by the mirror and the converter. Finally, the camera is covered on its sides by tiles of ${ }^{6} \mathrm{LiF}$ polymers and lead bricks to shield it against neutrons and gammas. A complete description of the set-up can be found in Ref. [6].

\subsection{Spatial resolution and acquisition time}

The spatial resolution is essentially degraded at first by the divergence of the neutron beam and secondly by the neutron to visible light conversion process in the scintillator screen. Different scintillators have been tested [7] and the best solution in terms of spatial resolution versus conversion efficiency was obtained with a $100 \mu \mathrm{m}$ thick $\mathrm{ZnS}(\mathrm{Ag}) /{ }^{6} \mathrm{LiF}$ screen from Appscintech [8]: when the sample is as close as possible to the screen, the spatial resolution of a radiograph is $0.24 \mathrm{~mm}$. The divergence of the neutron beam degrades linearly the resolution with the distance between the sample and the conversion screen: at $2.5 \mathrm{~cm}$, the resolution is already $0.5 \mathrm{~mm}$ and at $5 \mathrm{~cm}$, it is $0.87 \mathrm{~mm}$. For that reason, the distance is set at minimum before scanning a new sample. The typical time to acquire a $512 \times 640$ image covering the field of view of $2.7 \mathrm{~cm} \times 3.4 \mathrm{~cm}$ is about $2 \mathrm{~s}$ with the $100 \mu \mathrm{m}$ converter screen. Including the time to turn the sample, a complete tomography of a sample can be made in less than an hour.

An attempt was made to reduce the divergence by the use of Soller collimator [9]. A Soller collimator consists of vertical (or horizontal) blades coated with neutron absorbing material. We tested a $\left(\frac{1}{3}\right)^{\circ}$ divergent collimator and compared with the situation with no collimator installed. The spatial resolution is improved and the divergence calculated from the spatial resolution agrees with the divergence of 20'. However, there are many drawbacks that made its use inconvenient. (1) The distribution of neutrons after the collimator is highly perturbed: one sees residual effects of absorption in the vertical blades fading with distance to the collimator. (2) There is an increase of white spots created by gamma-rays emitted by the Soller. (3) The collimators are $30 \mathrm{~cm}$ long and two of them have to be used to effectively reduce the divergence in both directions.

\subsection{Examples of cold neutron tomography}

The cold neutron station at SINQ was used to perform many tomographies in various fields: space industry, nuclear industry, geology, biology, dentistry, etc. Fig. 1 shows for example the 3D reconstruction of a flower. Putting this flower in a lead container with 2-cm-thick wall would increase the acquisition time but the result would not change because lead is quasi-transparent compared to hydrogen. In the nuclear industry, this property can be used to analyze samples within their lead container: usually one needs the surface and volume of the sample without removing it from the lead shielding and this is done easily with neutron tomography [7]. In industry, the nondestructive property of tomography prevails in the case of quality control: one can analyze the 3D reconstruction of an object and extract parts of the artificial object. This property was tested for space industry [7] to inspect rubber rings (large hydrogen concentration) in a pyrotechnic cutter. Fig. 2 shows the results of a tomography of a standard spark plug.

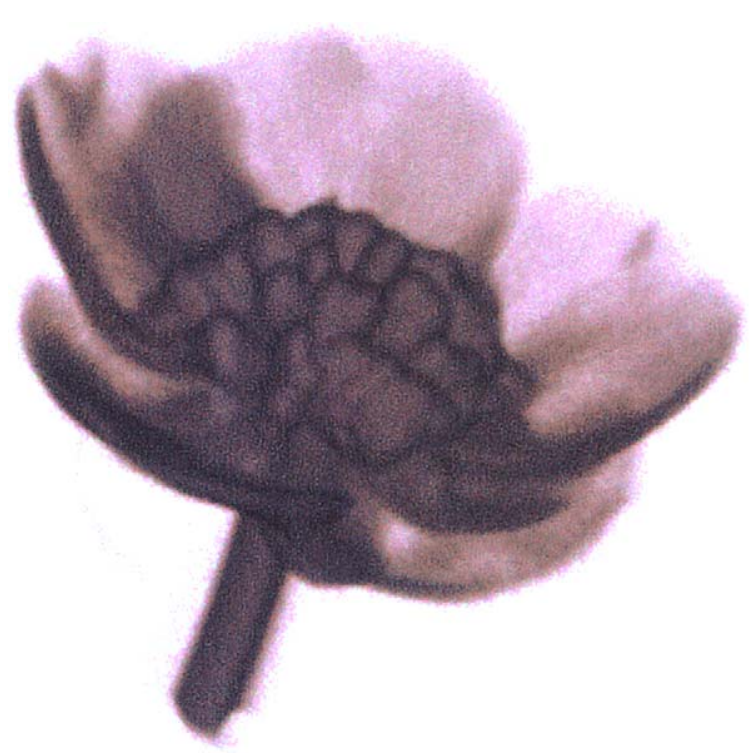

Fig. 1. Tomography of a buttercup is an example of the sensitivity of neutron to hydrogen in water. 

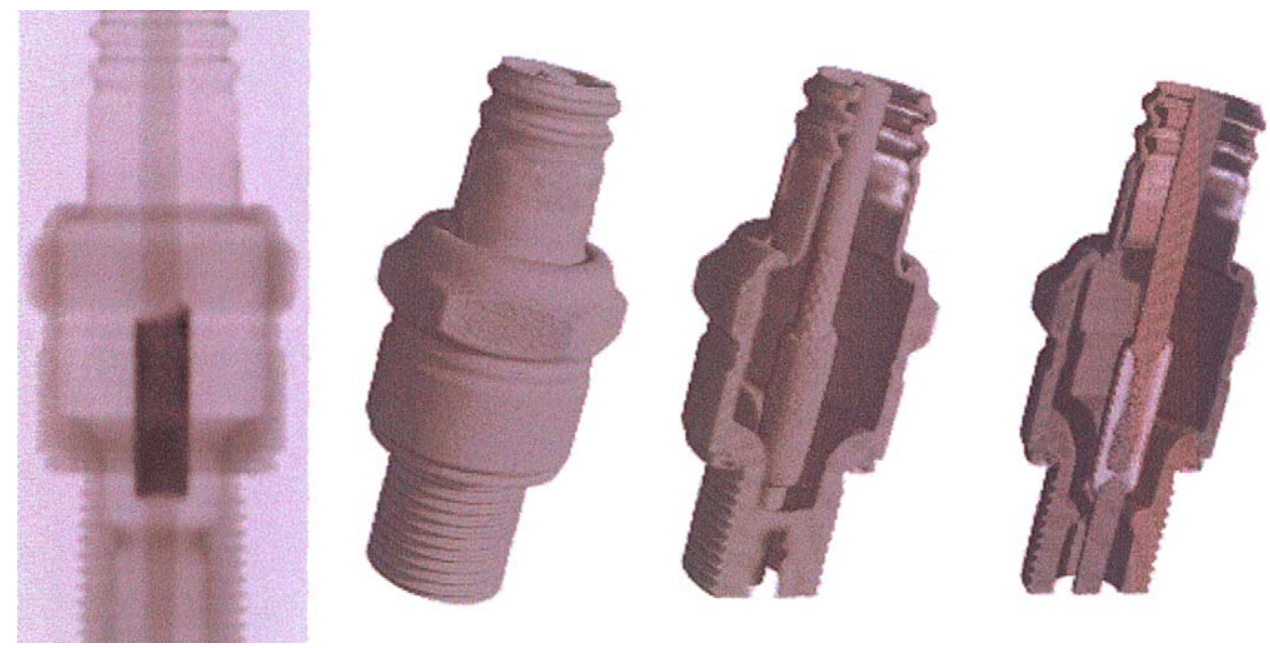

Fig. 2. Radiograph of a spark plug, the reconstructed 3D object and cuts in this virtual object.

\subsection{Set-up with velocity selector and dichromatic radiography}

A neutron velocity selector is a high-speed rotor, which is transparent only for neutrons that manage to pass between the twisted lamellae in a time interval defined by the rotation speed of the selector. This way, neutrons in a certain wavelength range are selected. We temporarily installed a velocity selector from Astrium $\mathrm{GmbH}$ before the detection system. With it we could choose wavelengths between 2.5 and $10.5 \AA$. The use of a velocity selector has two advantages. At first, the spatial resolution is improved at low wavelengths because the neutron divergence due to the reflection in the neutron beam guide is proportional to the wavelength. Secondly, it permits the performance of dichromatic radiography and tomography as explained in introduction. On the other hand, the selection of a wavelength decreases significantly the neutron flux and one has to choose a quite large wavelength width to keep a reasonable beam intensity. For example, the acquisition time at $2.7 \AA$ with a wavelength width of $30 \%$ was $80 \mathrm{~s}$ instead of $2 \mathrm{~s}$ for the white beam. This reduction in intensity prohibits the systematic use of a velocity selector for tomography at PSI but nice results were achieved in radiography and are detailed in Ref. [10].

\section{Installation at FRM-II and outlook}

In the near future, the cold neutron tomographic station will be installed at the new research reactor FRM-II in Garching close to Munich (Germany). At the time of writing, the neutron guide is under design. It should provide a neutron flux not less than $10^{9} \mathrm{n} / \mathrm{s} / \mathrm{cm}^{2}$, i.e. about 6 times more the one we had at SINQ. The neutron beam size at the exit of the guide should be about $5 \mathrm{~cm} \times 11.5 \mathrm{~cm}$ (i.e. $5.7 \times$ the area at SINQ) and will allow scanning of large samples. A neutron velocity selector will be available on this beam guide and will permit the performance of dichromatic tomographies in a reasonable time.

Compared to SINQ, we will install three stations at the same position: one for cold tomography, one for Prompt Gamma Activation Analysis (PGAA) and one for nuclear spectroscopy. All these installations will be on separate tables mounted on rails, so that one can move the desired station in front of the beam guide without dismounting a set-up.

At PSI, the main activity of PGAA was to perform elemental analysis of a sample totally irradiated with neutrons or with neutrons focused on a small part of the sample using a neutron lens [11]. It was possible at PSI to study the 2-D spatial distribution of elements in a sample by moving the sample in front of the neutron lens and it was done 
in Ref. [12] for a rock coming from the natural fossil reactor of Oklo but the required acquisition time (about an hour per position) forbids performing such study systematically. With the higher flux at FRM-II, this kind of analysis can be done faster and the results complement the one obtained with a standard neutron tomography.

\section{References}

[1] E.H. Lehmann, P. Vontobel, B. Schillinger, T. Bücherl, S. Baechler, J. Jolie, W. Treimer, R. Rosa, G. Bayon, S. Legoupil, S. Körner, V. Micherov, M. Balasko, in: Proceedings of the 15th World Conference on NonDestructive Testing, Rome, Italy, October 15-21, 2000, Available from http://www.ndt.net/article/wcndt00/ papers/idn804/idn804.htm.

[2] Th. Materna, J. Jolie, W. Mondelaers, B. Masschaele, V. Honkimäki, A. Koch, Th. Tschentscher, J. Synchrotron Radiat. 6 (1999) 1059.
[3] G.S. Bauer, Nucl. Instr. and Meth. B 139 (1998) 65.

[4] See http://neutra.web.psi.ch.

[5] See http://ncr.web.psi.ch.

[6] S. Baechler, B. Masschaele, P. Cauwels, M. Dierick, J. Jolie, T. Materna, W. Mondelaers, Nucl. Instr. and Meth. A 481 (2002) 397.

[7] S. Baechler, N. Kardjilov, M. Dierick, J. Jolie, G. Kühne, E. Lehmann, T. Materna, Nucl. Instr. and Meth. A 491 (2002) 481.

[8] General specifications of the screen are available on http:// www.appscintech.com.

[9] Th. Materna, S. Baechler, G. Kühne, R. Ene, E. Lehmann, M. Dierick, COST 524 STSM Report 15 May-15 June 2001, Available from http://ncr.web.psi.ch.

[10] N. Kardjilov, S. Baechler, M. Bastürk, M. Dierick, J. Jolie, E. Lehmann, T. Materna, B. Schillinger, P. Vontobel, Nucl. Instr. and Meth. A 501 (2003) 536.

[11] S. Baechler, P. Kudejova, J. Jolie, J.-L. Schenker, J. Radioanal. Nucl. Chem. 256 (2003) 239.

[12] S. Baechler, M. Crittin, J. Kern, T. Materna, P. Cauwels, B. Masschaele, W. Mondelaers, H.U. Johner, V. Honkimäki, M. Piboule, J. Radioanal. Nucl. Chem. 250 (2001) 39. 